\title{
Probing ice-nucleation processes on the molecular level using second harmonic generation spectroscopy
}

\author{
A. Abdelmonem ${ }^{1}$, J. Lützenkirchen ${ }^{2}$, and T. Leisner ${ }^{1}$ \\ ${ }^{1}$ Institute of Meteorology and Climate Research - Atmospheric Aerosol Research (IMKAAF), \\ Karlsruhe Institute of Technology (KIT), 76344 Eggenstein-Leopoldshafen, Germany \\ ${ }^{2}$ Institute of Nuclear Waste Disposal (INE), Karlsruhe Institute of Technology (KIT), \\ 76344 Eggenstein-Leopoldshafen, Germany
}

Correspondence to: A. Abdelmonem (ahmed.abdelmonem@kit.edu)

Received: 23 February 2015 - Published in Atmos. Meas. Tech. Discuss.: 22 May 2015

Revised: 26 July 2015 - Accepted: 2 August 2015 - Published: 27 August 2015

\begin{abstract}
We present and characterize a novel setup to apply second harmonic generation (SHG) spectroscopy in total internal reflection geometry (TIR) to heterogeneous freezing research. It allows to monitor the evolution of water structuring at solid surfaces at low temperatures prior to heterogeneous ice nucleation. Apart from the possibility of investigating temperature dependence, a major novelty in our setup is the ability of measuring sheet-like samples in TIR geometry in a direct way. As a main experimental result, we find that our method can discriminate between good and poor ice nucleating surfaces. While at the sapphire basal plane, which is known to be a poor ice nucleator, no structural rearrangement of the water molecules is found prior to freezing, the basal plane surface of mica, an analogue to ice active mineral dust surfaces, exhibits a strong change in the nonlinear optical properties at temperatures well above the freezing transition. This is interpreted as a pre-activation, i.e. an increase in the local ordering of the interfacial water which is expected to facilitate the crystallization of ice at the surface. The results are in line with recent predictions by molecular dynamics simulations on a similar system.
\end{abstract}

\section{Introduction}

Immersion freezing, i.e. the formation of ice on the surface of an insoluble aerosol particle (the ice nucleating particle, INP) in a supercooled droplet of water or an aqueous solution is the most important mode for formation of atmospheric ice (Pruppacher and Klett, 1997). It facilitates the initiation of precipitation and is largely responsible for the formation of cirrus clouds, which modify the global energy budget (Zhang et al., 1999). Despite its importance, little is known about the molecular structure of the critical ice nucleus and how it is stabilized by the interaction with aerosol surfaces.

Atmospheric aerosols can enhance heterogeneous Ice Nucleation (IN) in the deposition, contact and immersion mode. In the latter case, an insoluble aerosol particle immersed within a droplet of supercooled water or aqueous solution eventually leads to the formation of ice well above the temperature of homogeneous freezing (Pruppacher and Klett, 1997). Immersion freezing on mineral dust particles or primary biological particles is the dominant mode of heterogeneous freezing in the atmosphere (Korolev and Isaac, 2003; Pruppacher and Klett, 1997). Experimental immersion freezing studies of heterogeneous IN show a wide variability in behavior among the different atmospheric particles that are considered relevant (see Hoose and Möhler, (2012) and references therein).

It remains elusive which physical, structural and chemical characteristics of the INPs account for this variability and which aspects are dominant (Anim-Danso et al., 2013a; Conrad et al., 2005; Hu and Michaelides, 2007). Recent Molecular Dynamic (MD) simulations on heterogeneous IN of water on carbon surfaces suggest that good ice nucleators induce layering of liquid water at the surface (Lupi et al., 2014; Lupi and Molinero, 2014). Those authors proposed that such layering of water enhances the local ordering of the first water layer in contact with the surface. So far, no classification of ice nucleation abilities based on the chem- 
ical or physical properties of a potential ice nucleator has been published and hardly any laboratory based information is available neither on the molecular structure of the surface active sites and its role in the IN process nor on the molecular structure of the critical germ of water. Various methods have been employed to study heterogeneous IN, e.g. infra-red spectroscopy (Richardson et al., 2007; Sadtchenko and Ewing, 2011). Following this, one promising approach to elucidate these structures on the molecular level is nonlinear optical (NLO) spectroscopy. Second Harmonic Generation (SHG) and Sum Frequency Generation (SFG) are powerful surface sensitive spectroscopic tools for studying molecules near surfaces and at interfaces (Shen, 1989a). The amplitude and polarization of the generated field, as a function of the polarization of the incident fields, carries information on the orientation of the interfacial molecules (Abdelmonem, 2008; Jang et al., 2013; Rao et al., 2003; Zhuang et al., 1999).

SHG can provide insight into the interfacial molecular properties at an interface between two isotropic media. Its response relates to the overall arrangements of the water (or more general interfacial) entities (Fordyce et al., 2001; Goh et al., 1988; Luca et al., 1995). The resulting signal arises from the surface dipole contribution as well as from some contribution of higher-order terms from the bulk, the electric quadrupole and the magnetic dipole terms. Shen's group demonstrated systematically in the early 1980s that surface SHG can be treated as radiation from a nonlinear polarization sheet induced by an incoming wave at the surface, besides comparable quadrupole contributions (see Shen, (1989b) and other references from Shen therein). The dipolar part strongly depends on the absolute orientation of the water molecules (which belong to $\mathrm{C}_{2 \mathrm{v}}$ symmetry), whereas the quadrupolar contribution weakly depends on this (Goh and Eisenthal, 1989; Goh et al., 1988). SFG on the other hand includes scanning over the different vibrational bands of the species at the interface. In case of water, one can observe the development of the so-called ice-like and liquid-like water components at 3200 and $3420 \mathrm{~cm}^{-1}$, respectively. Band positions and widths are related to the bonding-debonding states (Du et al., 1993; Richmond, 2001). This can yield a deeper insight into how the water molecules behave at the specific interface under cooling and allow more detailed information on the interfacial water molecules.

The structure of water molecules adsorbed on a mica surface has been previously studied at room temperature using SFG spectroscopy (Miranda et al., 1998). More recently Yang et al. (2011) employed SFG spectroscopy at the liquid water/mica interface to explore the role of sulfuric acid coatings on mineral dust particles in IN (Yang et al., 2011). They found that the signal emerging from ordered water structures on the mica surface decreased above a concentration of $0.5 \mathrm{M}$ of $\mathrm{H}_{2} \mathrm{SO}_{4}$ and disappeared above $5 \mathrm{M}$ of $\mathrm{H}_{2} \mathrm{SO}_{4}$. This was taken as indirect evidence that ordering of water at the INP is related to ice nucleation.
Mica (a layered clay mineral: $\mathrm{KAl}_{2}\left[\mathrm{AlSi}_{3} \mathrm{O}_{10}(\mathrm{OH})_{2}\right]$ ) is believed to be among the most effective ice nucleating minerals in the deposition mode, at least as natural particles (Eastwood et al., 2008; Mason and Maybank, 1958). However, it could be different for the basal plane of mica. It was shown by (Holbrough et al., 2012; Campbell et al., 2013) that nucleation of some organic substances from vapor on mica surface (deposition mode) is enhanced only when producing a high-density of nanoscale features of the surface topography. This was not the case for ice nucleation from supercooled water droplets on the surface of mica (immersion mode) where they reported that freezing occurred close to the expected homogeneous freezing temperature and surface roughening had no significant effect (Campbell et al., 2015; Atkinson et al., 2013) showed that mica particles are not good ice nucleators in the immersion mode. On the other hand, (Steinke, 2013) using a cold stage setup showed that muscovite initiated heterogeneous ice nucleation at approximately $-23^{\circ} \mathrm{C}$ which is far above the homogenous freezing but much less than those of other ice-active mineral dusts. Sapphire $\left(\alpha-\mathrm{Al}_{2} \mathrm{O}_{3}\right)$ is considered to be a poor ice nucleator (Thomas, 2009; Richardson, 2006). A very recent study on IN processes using SFG focused on the structure of ice as well as freezing and melting transition temperatures for water in contact with the sapphire-c (basal) plane (Anim-Danso et al., 2013a). Those authors compared the amplitude of icelike and water-like peaks in the SFG spectra at different $\mathrm{pH}$ values just before and immediately after the freezing transition, but did not report the evolution of the SFG signal when approaching the freezing transition. One major focus was the influence of the surface charge in enhancing or delaying ice formation. They concluded that 1) even though the structures of water and ice are affected with $\mathrm{pH}$ the freezing point is independent of $\mathrm{pH}$ and 2) the heterogeneous freezing point was constant and about $-6^{\circ} \mathrm{C}$, which is a very high value for sapphire. Subsequent studies from the same group addressed the role of salt hydrates during freezing (Anim-Danso et al., 2013b; Zhang et al., 2014). So far no comparison between different surfaces has been published in order to evaluate their concomitant ability as ice nucleator.

In this work we demonstrate a novel setup which uses SHG, in total internal reflection (TIR) geometry, to probe water structuring at the interface upon supercooling. TIR measurements for a sheet-like sample (mica) are achieved by coupling the light from a prism-like sapphire single crystal using an appropriate index matching gel (IMG). The choice of the IMG material is explained in detail. An IN cell is integrated to the setup using a precise temperature-controlled (down to $-195^{\circ} \mathrm{C}$ ) cold stage. Two mineral surfaces are used as atmospheric aerosol analogues: sapphire $\left(\alpha-\mathrm{Al}_{2} \mathrm{O}_{3}\right)$ as a poor and mica as a good ice nucleating minerals. The evolution of water structuring, upon supercooling on the surface of mica is compared to that at the surface of sapphire. A sapphire prism was used as a substrate in sapphire/water exper- 
iments and as an optical-coupler for mica substrates in the $\mathrm{mica} /$ water experiments.

The focus of this article is to demonstrate the usefulness of the technique and to present first results on temperature dependent structural changes of water on the surfaces of sapphire and mica on cooling and discuss the findings in the light of the IN abilities of these surfaces.

\section{Experimental}

\subsection{Materials and setup}

All experiments are carried out using MilliQ water $(18.2 \mathrm{M} \Omega \cdot \mathrm{cm})$. The total organic content in this water is below $4 \mathrm{ppb}$. The sapphire prisms are obtained from Victor Kyburz AG, Safnern, Switzerland. The roughness of the sapphire surface is $1.5 \mathrm{~nm}$ and the flatness is lambda/4. Mica samples (Muscovite $\mathrm{KAl}_{2}\left[\mathrm{AlSi}_{3} \mathrm{O}_{10}(\mathrm{OH})_{2}\right]$ ) are obtained from Plano $\mathrm{GmbH}$, Germany. The mica samples are freshly cleaved in air. Both minerals used here expose their basal-planes and are probed in TIR geometry. The contact angle of the neat sapphire-c is $30^{\circ}$. The freshly cleaved mica shows a wetting surface (on which water was visually spreading). The contact angle measurements were carried out by the drop method for sapphire. Changes in the amount of dissolved gases are assumed to be minimal since the cell is closed. Furthermore, a possible effect of dissolved gas on the ice-nucleation behavior would be the same for both surfaces so that this is unlikely to affect the outcome.

For our experiments, we use a two-beam-SHG setup in TIR geometry (c.f. Fig. 1). A sapphire prism is used either directly as a substrate exposing the surface of interest or as an optical coupler to the surface of thin mica substrates. Both substrates ultimately expose their basal-planes to liquid water or water vapor. To be able to study mica, an index matching gel (IMG) is used to fix the mica sample on the hypotenuse of the sapphire prism (see details below).

As a further novelty, the setup is integrated into a temperature-controlled environmental chamber to study temperature-dependent signals. We used a commercially available cold-stage (Linkam model HFS-X350) and modified the housing to accommodate the SHG setup. Control software is developed to perform a predefined temperature profile and to collect the SHG signal, the reflectivities of the incident beams, and the temperature of the sapphire prism at two positions surrounding the point of laser interaction and of the fluid phase above the interface. Temperature profiles are repeated several times for each sample to test reproducibility. First they were applied to sapphire $/ \mathrm{N}_{2}$ and $\mathrm{mica} / \mathrm{N}_{2}$ (i.e. solid/gas) interfaces to assure that the change in the refractive indices of the substrates and IMG with temperature has no significant effect on the resulting SHG signal in the range of temperatures applied in this work. The SHG experiments are conducted using a femtosecond laser sys- tem (Solstice, Spectra Physics) with a fundamental beam of $800 \mathrm{~nm}$ wavelength, $3.5 \mathrm{~mJ}$ pulse energy, $\sim 80 \mathrm{fs}$ pulse width, $1 \mathrm{kHz}$ repetition rate, and a beam diameter of $1 \mathrm{~mm}$ at the interface. Only $30 \%$ of the laser output power is coupled to the setup. The TIR geometry provides an additional enhancement of the SHG signal if the incident beams are close to the critical angle (Abdelmonem, 2008). Instead of having a single fundamental beam incident on the interface, two incident beams at two different angles are allowed to overlap in space and time at the interface similar to SFG setups. This allows better separation between the background accompanying the fundamental beam and the signal from the probed interface (c.f. Fig. 1). The fundamental beam is split in two paths (B1 and B2) using a $50 \%$ beam splitter. The two split wave fronts of each pulse are spatially and temporally overlapped at the interface. Equal optical paths on both arms are achieved via a delay unit placed in the path of B1. The polarization direction of the incident beams is controlled by a half-wave plate followed by a cube polarizer. The generated signal is collected using a photomultiplier tube (PMT) placed beyond an optical system, which includes appropriate filters and a polarization analyzer. The experiments are carried out using two incident beams where B1 is adjusted to be at an angle slightly above the critical angle to avoid changes in signal due to the strong temperature dependence of the Fresnel coefficients at the critical angle. A fast video camera (Guppy F-036, Allied Vision Technology) is used to image macroscopic layers of water or ice on the crystal surface.

No vibrational states are excited here (fundamental at $800 \mathrm{~nm}$, SHG at $400 \mathrm{~nm}$ ). Non-resonant SHG provides orientational measurement of the non-straddle-type of interfacial water molecules (Zhang et al., 2005). We are working far from any resonant second-order susceptibility $\chi_{R}^{(2)}$, and the $\chi_{\mathrm{NR}}^{(3)}$ effect is ignored since we do not track ion adsorption at the interface (Ong et al., 1992). As mentioned above, the sum over the surface dipole moments is structure-dependent, the higher the ordering of the molecules the higher the overall dipole response. Two polarization combinations are required to describe the water system which has $\mathrm{C}_{2 \mathrm{v}}$ symmetry. We denote by $\mathrm{P}$ or $\mathrm{S}$ a polarization parallel or perpendicular to the plane of incidence, respectively. PPP polarization combination corresponds to P-SHG, P-B2 and P-B1 and SSP polarization combination corresponds to S-SHG, S-B2 and P-B1. The stronger the signal of either SSP or PPP, the more pronounced is the ordering of the molecules, while the ratio of SSP/PPP determines the average orientation of the interfacial (water) molecules (Abdelmonem, 2008). In this work we use PPP only to qualitatively follow the structural change of water molecules.

The substrate of interest is sealed to a circular opening of diameter $8 \mathrm{~mm}$ in a Teflon cell which can be filled with water or humid air. One of the two prism sides perpendicular to the hypotenuse is mounted on the cold stage. The temperatures of sample top, sample bottom and water (or air) inside the 


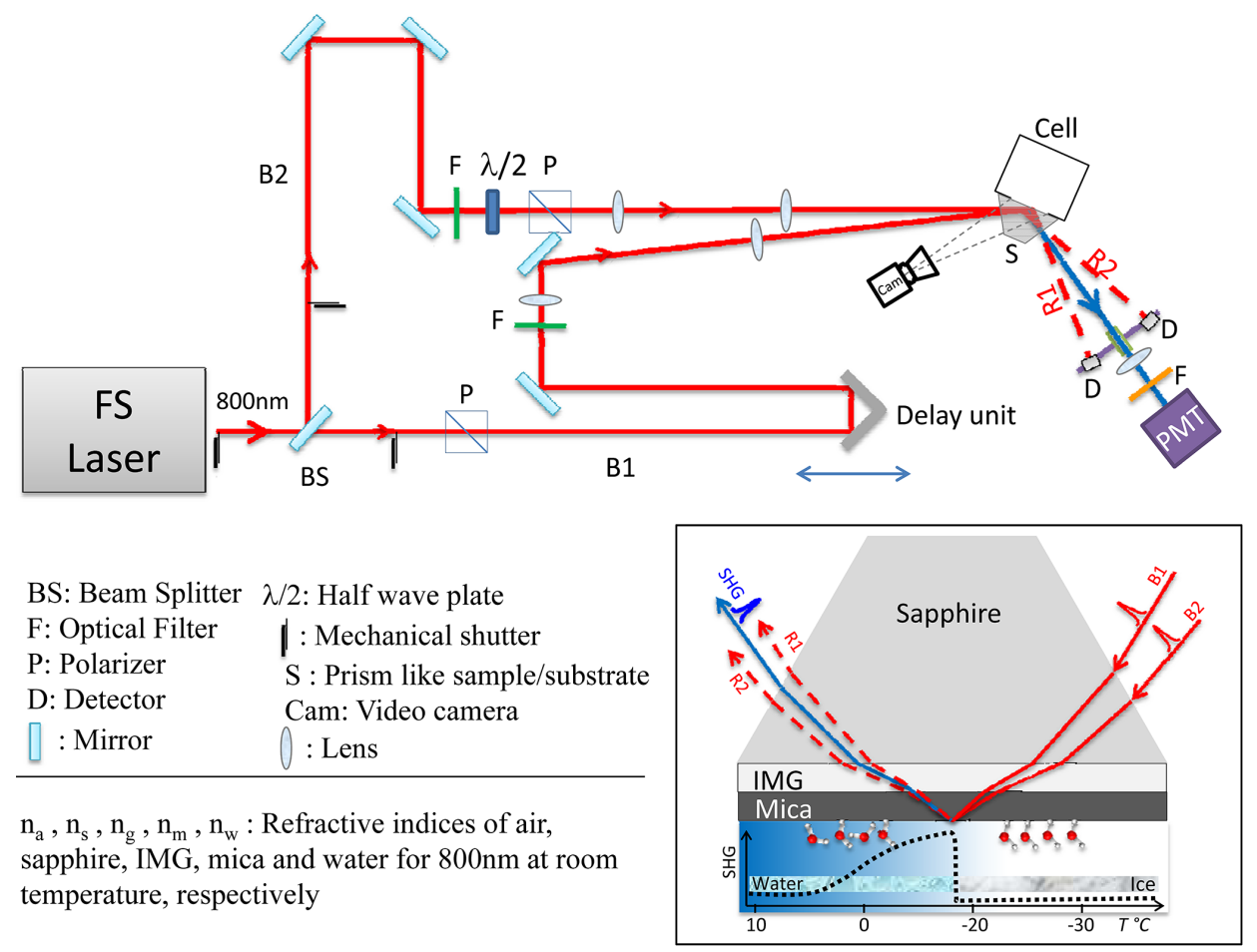

Figure 1. A schematic presentation of the SHG setup used in this study. Inset shows the sample geometry. See text for detailed explanations.

cell are recorded using four-wire-Pt100 elements. However, we are not interested here in the exact onset conditions of freezing, but rather study the qualitative behavior of water molecules during supercooling.

It is impossible to achieve the desired TIR geometry using substrates that are only available as plate-like samples as mica. Mica has a refractive index $\left(n_{\mathrm{m}}=1.564\right)$ lower than that of sapphire $\left(n_{\mathrm{s}}=1.7522\right)$ and higher than that of water $\left(n_{\mathrm{w}}=1.329\right)$, at $800 \mathrm{~nm}$. This allows access to the mica/water interface using the TIR geometry by applying an IMG between sapphire and mica, see inset Fig. 1. An IMG $\left(n_{\mathrm{g}}=1.45186\right)$ from Thorlabs is used in the present case. It satisfies the condition that there is no TIR at the sapphire/IMG or IMG/mica interface but at the mica/water interface. With our sample geometry, the appropriate incident angle of light from air $\left(\theta_{\mathrm{a}}\right)$ ranges from $\sim 7.1$ to $\sim 18.9^{\circ}$ (see Sect. 2.2 for details).

\subsection{Selection of optimum IMG}

For better beam coupling (i.e. larger acceptance angle), the refractive index of the IMG can be optimized to simultaneously maximize the critical angle of TIR at both sapphire/IMG and IMG/mica interfaces. We find that the optimum value for our system is $n_{\mathrm{g}} \geq n_{\mathrm{m}}$. The reflectivities at the sapphire/IMG, IMG/mica and mica/water interfaces of a P-polarized light as a function of the incident angle from air $\left(\theta_{\mathrm{a}}\right)$ are plotted in Fig. 2. A similar treatment is possible

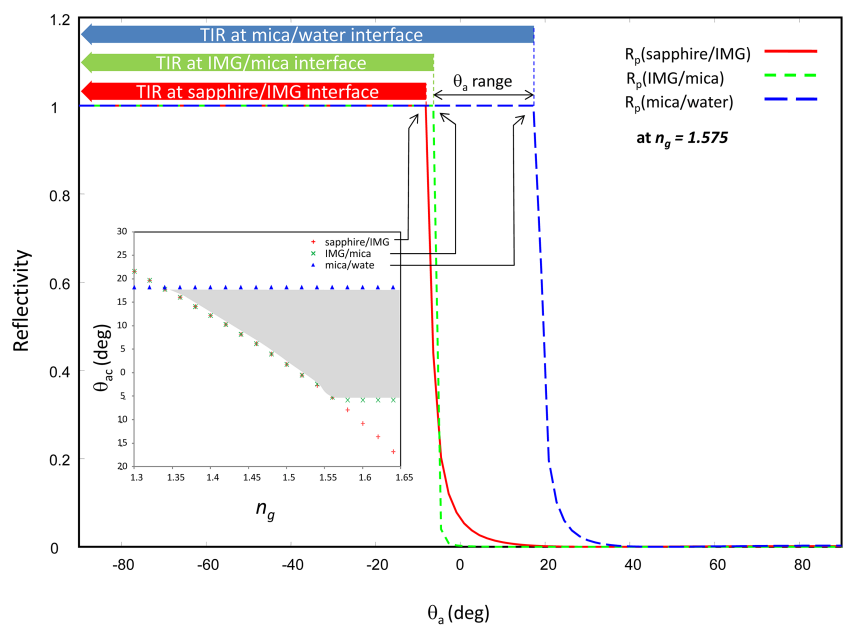

Figure 2. Reflectivities of a P-polarized (Rp) light as a function of the incident angle from air $\left(\theta_{\mathrm{a}}\right)$ at the different interfaces of inset Fig. 1. The reflectivities at sapphire/IMG, IMG/mica and mica/water interfaces are represented by the soli-red, dash-green and long-dash-blue lines respectively. An arbitrary value of $n_{\mathrm{g}}=$ 1.575 is chosen. The points of drop of the reflectivity for the three interfaces as a function of $n_{\mathrm{g}}$ are plotted on the inset. $\theta_{\mathrm{ac}}$ is the lower threshold of $\theta_{\mathrm{a}}$ for the sapphire/IMG and IMG/mica interfaces and the higher threshold of $\theta_{\mathrm{a}}$ for mica/water interface. The shaded area is the range of allowed incident angles from air at any $n_{\mathrm{g}}$. 

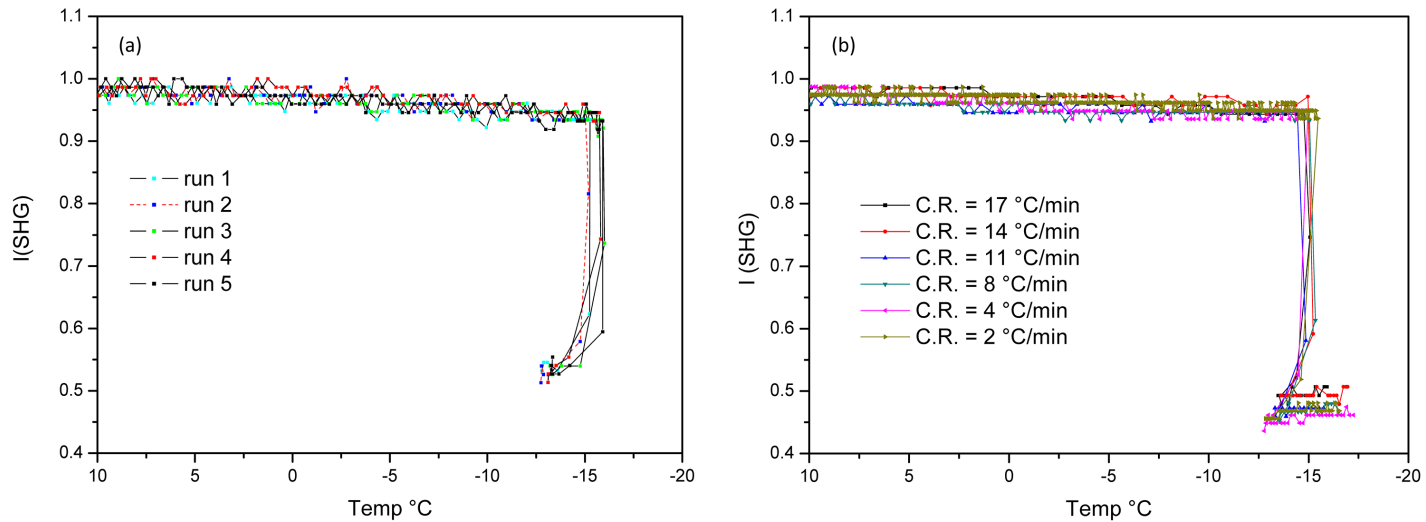

Figure 3. SHG at sapphire/water-ice interface as a function of temperature during cooling. (a) The same cooling profile of $-8^{\circ} \mathrm{C}$ min ${ }^{-1}$ cooling rate is repeated 5 times. (b) Different cooling rates.

for S-polarized light. An arbitrary value $\left(n_{\mathrm{g}}=1.575\right)$ is used for the illustrative calculations shown on this plot. The suitable range of $\theta_{\mathrm{a}}$ should have a minimum higher than those corresponding to the critical angles of TIR at sapphire/IMG and IMG/mica interfaces (the points of drop of the solid-red and dashed-green curves) and a maximum lower than that for mica/water interface (the point of drop of the long-dashedblue curve). At lower angles the beam is totally reflected at the sapphire/IMG or IMG/mica interface and at higher angles the beam violates the TIR condition at the mica/water interface. The inset in Fig. 2 shows the points of drop of the reflectivity for the three interfaces (lower and higher thresholds of $\theta_{\mathrm{a}}$ ) as a function of $n_{\mathrm{g}}$. The shaded area is the range of suitable incident angles from air at any $n_{\mathrm{g}}$. In our study we used an IMG with $n_{\mathrm{g}}=1.45186$. The corresponding appropriate incident angle of light from air $\left(\theta_{\mathrm{a}}\right)$ ranges from $\sim 7.1$ to $\sim 18.9^{\circ}$.

\section{Results and discussion}

We explore immersion freezing, i.e. water molecule ordering and ice formation at the interface between the crystal and bulk water. For this purpose, the cell was filled with pure water and a cooling ramp is applied to the whole system. Figure $3 \mathrm{a}$ shows the change in the SHG signal at the sapphire/water interface for a cooling profile from $10^{\circ} \mathrm{C}$ to about $-15^{\circ} \mathrm{C}$. The cooling profile (cooling rate: $8^{\circ} \mathrm{C} \mathrm{min}^{-1}$ ) is repeated five times to confirm the reproducibility of the result. The SHG signal shows a slight decrease with temperature before the phase change. A step is observed at the phase transition (ice/sapphire interface). Other cooling profiles with different cooling rates $\left(17,14,11,8,4\right.$ and $\left.2{ }^{\circ} \mathrm{C} \mathrm{min}^{-1}\right)$ in the same temperature range did not lead to different results (see Fig. 3b). The slight change in the SHG before the phase change could be due to temperature dependence of the optical properties of water and sapphire. Similar observations were reported for the range between 20 and $70^{\circ} \mathrm{C}$ (Zhang

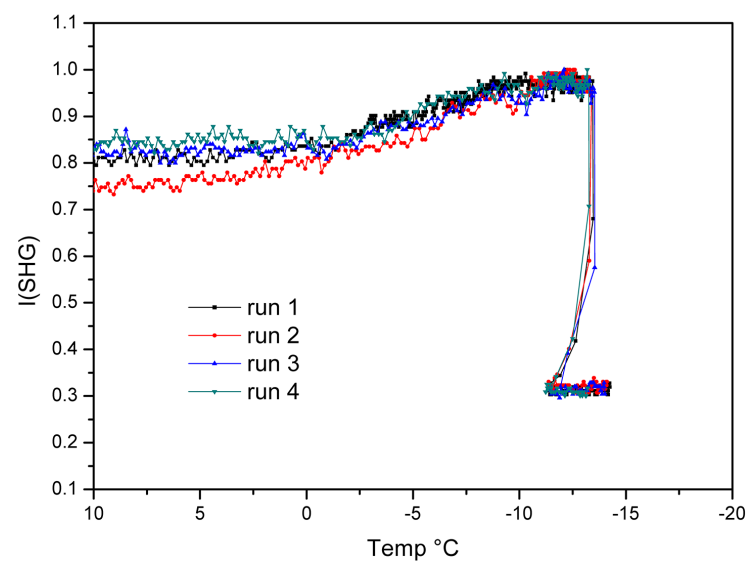

Figure 4. SHG at mica/water-ice interface as a function of temperature during cooling at a cooling rate of $-2{ }^{\circ} \mathrm{C} \min ^{-1}$.

et al., 2005). The slight temperature increase after the phase change is due to the release of latent heat. The latent heat is related to the amount of water frozen at the interface which has the same contact area in both cases (cell opening), same sensor positions, same cooling source. There can be small differences due to the difference in material properties involved in the heat transfer from the interface to the sensors (like substrate itself, and IMG in case of mica) and the exact positions of the temperature sensors. The vertical drop corresponds to the difference between signals of water/substrate and ice/substrate interfaces.

In contrast, Fig. 4 shows the evolution of the SHG signal generated at the mica/water interface for a cooling profile in the same range of that of sapphire/water interface. The cooling profile is repeated four times at the same rate $\left(2{ }^{\circ} \mathrm{C} \mathrm{min}^{-1}\right)$. Well before the phase transition, the SHG signal is rising. This observation suggests an enhancement in the local ordering of the interfacial water in contact with the surface upon supercooling (a pre-activation). This pro- 
cess in turn is expected to facilitate ice crystallization at the surface. Water molecules form strong hydrogen bonds with their neighbors upon cooling and gradually start to fit into positions that resemble the hexagonal packing achieved upon freezing (Smith, 2004). Here, we find indications that this hexagonal packing is probably facilitated by the mica surface since it induces pre-packing in the liquid phase. Although SHG is monolayer sensitive, secondary layers may have an indirect effect on the signal by affecting this first layer. However, this would not change our interpretation that the surface can induce water structuring upon temperature change. Recently it was reported that freezing at air/water interfaces does not proceed via the hexagonal basal face (Shultz et al., 2014). Even though the mica samples were freshly cleaved before each experiment small variations were observed at high temperatures (see Fig. 4), but the trend was always the same and different from that observed consistently with sapphire. While it is the focus of this paper to demonstrate the ability of this technique to observe variations at the interface of different aerosol surfaces, we plan to investigate the origin of the observed mica behavior in a separate study. Figure 4 shows the typically observed relation.

From the present results we cannot know the precise position at which the ice nucleation starts. Once the critical germ forms at any point, freezing propagates along the surface at a rate of about $0.1 \mathrm{~m} \mathrm{~s}^{-1}$ (Rauschenberger et al., 2013; Shibkov et al., 2003, 2005). The video recording could determine that the ice phase was nucleated at the interface between the crystal surface and the cell opening and subsequently propagated through our optically probed region. This explains the freezing obtained at temperatures much higher than those expected for the two selected minerals and confirms our concerns about the freezing point published by (Anim-Danso et al., 2013a) on water freezing at the sapphire surface. We believe that even in their case ice nucleation started somewhere else but not the probed water/sapphire interface especially since they were cooling the whole cell while we cool the sample directly. Avoiding ice nucleation at the joint point between the cell and the surface is impossible with the cell designs presently available. However, experimentally we show that the technique is able to resolve a pre-activation induced by the surface of a good ice nucleator. Our results agree with the theoretical predictions by Lupi et al. (2014) which show that good ice nucleators induce layering of the liquid water which in turn enhances the local ordering of the first water layer in contact with the surface (Lupi et al., 2014; Lupi and Molinero, 2014).

\section{Conclusions}

We have demonstrated the capability of a novel system to investigate heterogeneous ice nucleation on the molecular level before, during and after the phase change. Sheet-like sample could be studied in TIR geometry by applying a suitable IMG between the sample and a prism-like optical coupler. The selection of an appropriate IMG for such purposes is discussed. Immersion freezing was studied on surfaces of two different minerals that are known to be poor and good ice nucleators, respectively. Sapphire, as a model surface of a poor IN agent, showed no influence on the water structure at its surface upon cooling down to the point of freezing. However, mica, as a good IN agent, induced local ordering of the first water layer in contact with the surface which is represented by the enhancement of the SHG upon cooling. This strong ordering of water molecules is expected to facilitate the crystallization of ice at the surface. This fits well with the recent theoretical findings on graphite (Lupi et al., 2014). To our knowledge, this is the first experimental confirmation that heterogeneous ice crystallization on a surface is related to the degree of order induced by this surface to supercooled water. For more specific characterization of ice nucleation at mica/water interfaces and similar systems, our setup should be enhanced to allow SFG studies to gain more direct orientational and dynamic information on the interfacial water molecules by tracking different specific vibrational bands, such as those of liquid-like and ice-like contributions. This will allow probing the structuring of water molecules and correlating ice nucleation efficiency to the structural and chemical characteristics of the surface. Further experiments on mica/water interface are essential to understand its behavior in bare aquatic solutions. To overcome the extrinsic ice nucleation a special cell is required and will be considered in the future works.

Acknowledgements. The fund for building the SHG setup including the temperature controlled cold stage was received from the competence area "Earth and Environment" - KIT through a start-up budget (2012). The work was initiated with a start-up fund from the KIT and is currently funded by the German Research Foundation (DFG, AB 604/1-1).

The article processing charges for this open-access publication were covered by a Research Centre of the Helmholtz Association.

Edited by: F. Pope

\section{References}

Abdelmonem, A.: Nonlinear optical spectroscopy at the Liquid/Solid- interface, 1.0, Naturwissenschaften, Mathematik und Informatik, Ruprecht-Karls-Universität, Heidelberg, 16-38, 2008.

Anim-Danso, E., Zhang, Y., Alizadeh, A., and Dhinojwala, A.: Freezing of water next to solid surfaces probed by infraredvisible sum frequency generation spectroscopy, Am. Chem. J., 135, 2734-2740, 2013a.

Anim-Danso, E., Zhang, Y., and Dhinojwala, A.: Freezing and Melting of Salt Hydrates Next to Solid Surfaces Probed by Infrared-Visible Sum Frequency Generation Spectroscopy, Am. Chem. J., 135, 8496-8499, doi:10.1021/ja403437c, 2013b. 
Atkinson, J. D., Murray, B. J., Woodhouse, M. T., Whale, T. F., Baustian, K. J., Carslaw, K. S., Dobbie, S., O'Sullivan, D., and Malkin, T. L.: The importance of feldspar for ice nucleation by mineral dust in mixed-phase clouds, Nature, 498, 355-358, doi:10.1038/nature12278, 2013.

Campbell, J. M., Meldrum, F. C., and Christenson, H. K.: Characterization of Preferred Crystal Nucleation Sites on Mica Surfaces, Cryst. Growth Des., 13, 1915-1925, doi:10.1021/cg301715n, 2013.

Campbell, J. M., Meldrum, F. C., and Christenson, H. K.: Is Ice Nucleation from Supercooled Water Insensitive to Surface Roughness?, The Journal of Physical Chemistry C, 119, 1164-1169, doi:10.1021/jp5113729, 2015.

Conrad, P., Ewing, G. E., Karlinsey, R. L., and Sadtchenko, V.: Ice nucleation on BaF2(111), J. Chem. Phys., 122, 064709, doi:10.1063/1.1844393, 2005.

Du, Q., Superfine, R., Freysz, E., and Shen, Y. R.: Vibrational spectroscopy of water at the vapor/water interface, Phys. Rev. Lett., 70, 2313-2316, 1993.

Eastwood, M. L., Cremel, S., Gehrke, C., Girard, E., and Bertram, A. K.: Ice nucleation on mineral dust particles: Onset conditions, nucleation rates and contact angles, J. Geophys. Res.: Atmos., 113, D22203, doi:10.1029/2008jd010639, 2008.

Fordyce, A. J., Bullock, W. J., Timson, A. J., Haslam, S., Spencer-Smith, R. D., Alexander, A., and Frey, J. G.: The temperature dependence of surface second-harmonic generation from the air-water interface, Mol. Phys., 99, 677-687, doi:10.1080/00268970010030022, 2001.

Goh, M. C. and Eisenthal, K. B.: The energetics of orientation at the liquid-vapor interface of water, Chem. Phys. Lett., 157, 101-104, doi:10.1016/0009-2614(89)87216-1, 1989.

Goh, M. C., Hicks, J. M., Kemnitz, K., Pinto, G. R., Heinz, T. F., Eisenthal, K. B., and Bhattacharyya, K.: Absolute orientation of water molecules at the neat water surface, J. Phys. Chem., 92, 5074-5075, doi:10.1021/j100329a003, 1988.

Holbrough, J. L., Campbell, J. M., Meldrum, F. C., and Christenson, H. K.: Topographical Control of Crystal Nucleation, Cryst. Growth Des., 12, 750-755, doi:10.1021/cg201084j, 2012.

Hoose, C. and Möhler, O.: Heterogeneous ice nucleation on atmospheric aerosols: a review of results from laboratory experiments, Atmos. Chem. Phys., 12, 9817-9854, doi:10.5194/acp-12-98172012, 2012.

$\mathrm{Hu}, \mathrm{X}$. L. and Michaelides, A.: Ice formation on kaolinite: Lattice match or amphoterism?, Surface Science, 601, 5378-5381, doi:10.1016/j.susc.2007.09.012, 2007.

Jang, J. H., Lydiatt, F., Lindsay, R., and Baldelli, S.: Quantitative Orientation Analysis by Sum Frequency Generation in the Presence of Near-Resonant Background Signal: Acetonitrile on Rutile TiO2 (110), J. Phys. Chem. A, 117, 6288-6302, doi:10.1021/jp401019p, 2013.

Korolev, A. and Isaac, G.: Phase transformation of mixedphase clouds, Q. J. Roy. Meteor. Soc., 129, 19-38, doi:10.1256/qj.01.203, 2003.

Luca, A. A. T., Hebert, P., Brevet, P. F., and Girault, H. H.: Surface second-harmonic generation at air/solvent and solvent/solvent interfaces, J. Chem. Soc., Faraday Trans., 91, 1763-1768, doi:10.1039/ft9959101763, 1995.
Lupi, L. and Molinero, V.: Does Hydrophilicity of Carbon Particles Improve Their Ice Nucleation Ability?, J. Phys. Chem. A, 118, 7330-7337, doi:10.1021/jp4118375, 2014.

Lupi, L., Hudait, A., and Molinero, V.: Heterogeneous Nucleation of Ice on Carbon Surfaces, Am. Chem. J., 136, 3156-3164, doi:10.1021/ja411507a, 2014.

Mason, B. J. and Maybank, J.: Ice-nucleating properties of some natural mineral dusts, Q. J. Roy. Meteor. Soc., 84, 235-241, doi:10.1002/qj.49708436104, 1958.

Miranda, P. B., Xu, L., Shen, Y. R., and Salmeron, M.: Icelike Water Monolayer Adsorbed on Mica at Room Temperature, Phys. Rev. Lett., 81, 5876-5879, 1998.

Ong, S., Zhao, X., and Eisenthal, K. B.: Polarization of water molecules at a charged interface: second harmonic studies of the silica/water interface, Chem. Phys. Lett., 191, 327-335, doi:10.1016/0009-2614(92)85309-X, 1992.

Pruppacher, H. R. and Klett, J. D.: Microphysics of clouds and precipitation, 2nd rev. and enl. ed., Atmospheric and oceanographic sciences library, 18, Kluwer Academic Publishers, Dordrecht, Boston, 954 pp., 1997.

Rao, Y., Tao, Y.-S., and Wang, H.-F.: Quantitative analysis of orientational order in the molecular monolayer by surface second harmonic generation, J. Chem. Phys., 119, 5226-5236, doi:10.1063/1.1597195, 2003

Rauschenberger, P., Criscione, A., Eisenschmidt, K., Kintea, D., Jakirlić, S., Tuković, Ž., Roisman, I. V., Weigand, B., and Tropea, C.: Comparative assessment of Volume-ofFluid and Level-Set methods by relevance to dendritic ice growth in supercooled water, Comput. Fluids, 79, 44-52, doi:10.1016/j.compfluid.2013.03.010, 2013.

Richardson, H. H., Hickman, Z. N., Thomas, A. C., Dendramis, K. A., Thayer, G. E., and Ewing, G. E: Freezing of water on alpha- $\mathrm{Al}_{2} \mathrm{O}_{3}$ surfaces, The 11th International Conference on the Physics and Chemistry of Ice, Bremerhaven, Germany 23-28 July 2006, p. 513, 2006.

Richardson, H., Thomas, A., Carlson, M., Kordesch, M., and Govorov, A.: Thermo-optical Responses of Nanoparticles: Melting of Ice and Nanocalorimetry Approach, J. Electron. Mater., 36, 1587-1593, doi:10.1007/s11664-007-0279-1, 2007.

Richmond, G.: Structure and bonding of molecules at aqueous surfaces, Annu. Rev. Phys. Chem., 52, 357-389, doi:10.1146/annurev.physchem.52.1.357, 2001.

Sadtchenko, V. and Ewing, G. E.: A new approach to the study of interfacial melting of ice: infrared spectroscopy, Can. J. Phys., 81, 333-341, doi:10.1139/p03-009, 2011.

Shen, Y. R.: Surface properties probed by second-harmonic and sum-frequency generation, Nature, 337, 519-525, 1989a.

Shen, Y. R.: Optical Second Harmonic Generation at Interfaces, Annu. Rev. Phys. Chem., 40, 327-350, doi:10.1146/annurev.pc.40.100189.001551, 1989b.

Shibkov, A. A., Golovin, Y. I., Zheltov, M. A., Korolev, A. A., and Leonov, A. A.: Morphology diagram of nonequilibrium patterns of ice crystals growing in supercooled water, Phys. A, 319, 6579, doi:10.1016/S0378-4371(02)01517-0, 2003.

Shibkov, A. A., Zheltov, M. A., Korolev, A. A., Kazakov, A. A., and Leonov, A. A.: Crossover from diffusion-limited to kineticslimited growth of ice crystals, J. Cryst. Growth, 285, 215-227, doi:10.1016/j.jcrysgro.2005.08.007, 2005. 
Shultz, M. J., Bisson, P. J., and Brumberg, A.: Best Face Forward: Crystal-Face Competition at the Ice-Water Interface, J. Phys. Chem. B, 118, 7972-7980, doi:10.1021/jp500956w, 2014.

Smith, R.: Conquering chemistry, 4th ed., McGraw-Hill, Sydney, 344 pp., 2004.

Steinke, I.: Ice nucleation properties of mineral dusts, The Faculty of Physics and Astronomy - Institute of Environmental Physics, Heidelberg University, Heidelberg, 174 pp., 2013.

Thomas, A. C.: Growth of Thin Film Water on $\alpha-\mathrm{Al}_{2} \mathrm{O}_{3}$ (0001) and its Implications for Ice Nucleation, Ohio University, 62-63, 2009.

Yang, Z., Bertram, A. K., and Chou, K. C.: Why Do Sulfuric Acid Coatings Influence the Ice Nucleation Properties of Mineral Dust Particles in the Atmosphere?, J. Phys. Chem. Lett., 2, 12321236, doi:10.1021/jz2003342, 2011.
Zhang, W.-K., Zheng, D.-S., Xu, Y.-Y., Bian, H.-T., Guo, Y., and Wang, H.-F.: Reconsideration of second-harmonic generation from isotropic liquid interface: Broken Kleinman symmetry of neat air/water interface from dipolar contribution, J. Chem. Phys., 123, 22471301-22471311, doi:10.1063/1.2136875, 2005.

Zhang, Y., Macke, A., and Albers, F.: Effect of crystal size spectrum and crystal shape on stratiform cirrus radiative forcing, Atmos. Res., 52, 59-75, doi:10.1016/S0169-8095(99)00026-5, 1999.

Zhang, Y., Anim-Danso, E., and Dhinojwala, A.: The Effect of a Solid Surface on the Segregation and Melting of Salt Hydrates, Am. Chem. J., 136, 14811-14820, doi:10.1021/ja5067866, 2014.

Zhuang, X., Miranda, P. B., Kim, D., and Shen, Y. R.: Mapping molecular orientation and conformation at interfaces by surface nonlinear optics, Phys. Rev. B, 59, 12632-12640, 1999. 